\title{
A germline or de novo mutation in two families with Gaucher disease: implications for recessive disorders
}

\author{
Hamid Saranjam ${ }^{1}$, Sameer S Chopra ${ }^{2,3}$, Harvey Levy ${ }^{3}$, Barbara K Stubblefield ${ }^{1}$, Emerson Maniwang ${ }^{1}$, \\ Ian J Cohen ${ }^{4,5}$, Hagit Baris ${ }^{4,5}$, Ellen Sidransky ${ }^{\star 1}$ and Nahid Tayebi ${ }^{1}$
}

Gaucher disease (GD) is an autosomal recessive storage disorder that most commonly results from the inheritance of one identifiable mutant glucocerebrosidase (GBA1) allele from each parent. Here, we report two cases of type 2 GD resulting from the inheritance of one identifiable paternal mutant allele and one allele that likely resulted from a maternal germline mutation. Germline mutations or mosiacism are not generally associated with autosomal recessive disorders. The probands from the two unrelated families had the same maternal mutation, leu444pro, that we propose resulted from a de novo maternal germline mutation occurring at this known 'hotspot' for mutation. This first report of a germline mutation for a common point mutation leu444pro (c.1448 T>C;p.leu483pro) in GD has significant implications for molecular diagnostics and genetic counseling in recessive disorders.

European Journal of Human Genetics (2013) 21, 115-117; doi:10.1038/ejhg.2012.105; published online 20 June 2012

Keywords: acute neuronopathic Gaucher disease; glucocerebrosidase; germline mutation; DNA mutational analysis; molecular diagnostic

\section{INTRODUCTION}

Gaucher disease (GD), the most common lysosomal storage disorder, results from the autosomal recessively inherited deficiency of glucocerebrosidase (GCase, EC 3.2.1.45). ${ }^{1,2}$ GD is subdivided into three types based on the presence and severity of CNS involvement. Type 2, acute neuronopathic GD, is the most severe form with early and progressive neurologic involvement, leading to death prenatally, in infancy or by early childhood. The gene encoding GCase (GBA1, MIM no. 606463) has a highly homologous pseudogene located $16 \mathrm{~kb}$ downstream on chromosome 1q21, sharing 96\% exonic sequence homology. This pseudogene has, over time, given rise to recombinant mutant alleles. ${ }^{3}$ Over 300 unique mutations have been identified in GBA1. Mutation leu444pro (c.1448 T > C;p.leu483pro) is a common mutant allele encountered in many different populations. ${ }^{4-10}$ The high frequency and worldwide distribution of the leu444pro mutation indicates that the site may be a 'mutational hotspot' for a $\mathrm{T} \rightarrow \mathrm{C}$ nucleotide transversion on c.1448 of GBA. This $\mathrm{T} \rightarrow \mathrm{C}$ substitution occurs both as a single point mutation and as part of a recombinant allele with other pseudogene sequence, further implicating this site as a mutational hotspot. ${ }^{11}$

Studies in sperm have made it possible to estimate the distribution and frequency of new human germline mutations at specific sites, providing evidence that premeiotic gonadal cells carrying a mutation might have a selective advantage, resulting in an increased likelihood of germline mosiacism or a new mutation in germline cells. ${ }^{12,13}$ This phenomenon may also contribute to the high frequency of the leu444pro mutation among different populations. We encountered two families, each with an affected child with type $2 \mathrm{GD}$, where the paternal mutant allele was identified in their respective fathers.
However, in both cases, the second mutant allele, leu444pro, was not detected in the mother. This prompted us to explore whether the leu444pro mutation might have resulted from a maternal germline mutation in these families. One other case of a presumed germline recombinant mutation in GD has been reported ${ }^{14}$ that includes the leu444pro alteration, but not as a point mutation.

\section{PATIENTS AND METHODS}

Family no. 1:

The proband, a female born at term to non-consanguineous parents of non-Jewish, European (German, British and Swedish) and Puerto Rican and Native American descent, had an early course complicated by stridor, copious secretions, poor feeding, gastroesophageal reflux, swallowing difficulties, ophthalmoplegia, paroxysmal apneic episodes, failure to thrive and developmental delay. At 5 months of age, a chest MRI revealed a vascular ring/double aortic arch that was surgically corrected at 7 months of age. A Nissen fundoplication and gastrostomy were performed at 8 months of age to address worsening reflux and persistent feeding difficulties. Laboratory findings demonstrated anemia and thrombocytopenia, and a bone marrow biopsy revealed lipid-laden macrophages characteristic of GD. A lysosomal enzyme panel demonstrated diminished GCase activity. The early onset of disease, coupled with apneic spells, ophthalmoplegia, stridor, swallowing difficulties, poor gross motor development and failure to thrive strongly suggested a diagnosis of type 2 GD. The patient died at 11 months of age.

Family no. 2:

This proband, born to a non-consanguineous Ashkenazi Jewish father and a Sephardic Jewish mother, has been described previously. ${ }^{15} \mathrm{He}$ was diagnosed at the age of 7 months after a bone marrow aspiration revealed Gaucher cells.

${ }^{1}$ Section on Molecular Neurogenetics, Medical Genetics Branch, National Human Genome Research Institute, NIH, Bethesda, MD, USA; ${ }^{2}$ Department of Medicine, Brigham and Women's Hospital, Harvard Medical School, Boston, MD, USA; ${ }^{3}$ Division of Genetics, Children's Hospital Boston, Harvard Medical School, Boston, MA, USA; ${ }^{4}$ Schneider Gaucher Clinic, The Rapheal Recanti Genetic Institute, Rabin Medical Center, Petah Tikva, Israel; ${ }^{5}$ Sackler Faculty of Medicine, Tel-Aviv University, Ramat Aviv, Israel

*Correspondence: Dr E Sidransky, Chief, Section on Molecular Neurogenetics, Medical Genetics Branch, National Human Genome Research Institute, NIH, Building 35, Room 1A213, 35 Convent Drive, MSC 3708, Bethesda, MD 20892-3708, USA. Tel: +1 3014510901 or +1 3014960373 ; Fax: +1 301402 6438; E-mail: sidranse@mail.nih.gov Received 1 December 2011; revised 6 March 2012; accepted 24 April 2012; published online 20 June 2012 
The diagnosis was confirmed by deficient GCase activity, and he died at 10 months secondary to respiratory failure.

\section{Genotyping:}

GBA1 gene was sequenced in genomic DNA samples from both probands and their family members. Genomic DNA was extracted from leukocytes, and/or fibroblast and buccal cells. The GBA1 gene was amplified from genomic DNA in three fragments encompassing exons $1-5,5-7$ and $8-11$ as previously described. ${ }^{16}$ Amplified fragments were purified using a QIAquick PCR Purification Kit (Qiagen, Santa Clarita, CA, USA), and Cycle sequencing was performed using the DyeTerminator Cycle Sequencing kit (Applied Biosystems, Foster City, CA, USA) with both forward and reverse primers, ${ }^{16}$ using an ABI Prism 3730XL DNA Analyzer (Applied Biosystems). The sequencing data was analyzed by SeqScape v2.6 using GeneBank NG_009783.1 as the reference sequence. To confirm the mutation, and exclude any chance of nucleotide variation at the primer site in the mother, a 700-bp amplified region of GBA1 surrounding the leu444pro mutation was digested with the restriction enzyme Nci I (New England Biolabs, Ipswich, MA, USA) as previously described. ${ }^{17}$ Long-range PCR of GBA also was performed on maternal DNA to rule out deletions or nucleotide variations at the primer sites. ${ }^{18}$

\section{Maternity testing}

DNA samples from the parents and probands in both families were sent to the DNA diagnostics Center (Fairfield, OH, USA). The probability of maternity was evaluated using 32 markers for Family no. 1 and with 16 markers for Family no. 2.

\section{Enzymatic activity and western blotting}

Enzyme activity was determined using a 4-methylumbelliferyl- $\beta$-D-glucopyranoside substrate (Sigma, St Louis, MO, USA). Western blot analysis was performed on fibroblast extracts from proband no. 1, her parents, and two controls as previously described. ${ }^{19,20}$

\section{RESULTS}

The genotype of proband no. 1 was thr323ile (c.1085C > T;p.thr362ile)/ leu444pro (c.1448 T > C;p.leu483pro; Figure 1a) based on genomic DNA sequencing data. Sequencing of paternal genomic DNA identified a heterozygous mutation, thr323ile, which was previously reported in a patient with type 1 GD. ${ }^{21}$ Surprisingly, no mutation was detected in the mother. The proband had a maternal half sibling, and genomic sequencing of her DNA also failed to demonstrate the leu444pro mutation. Prenatal testing of a subsequent pregnancy demonstrated that the fetus carried thr323ile, but not the leu444pro mutation. Evaluation of 32 different DNA markers in the parents and the child confirmed maternity with over $99.9 \%$ certainty. Leu444pro mosaicism was deemed unlikely, based upon documentation of normal GBA1 sequence in different maternal tissues, including blood, skin fibroblasts and buccal cells. Restriction digestion with the enzyme Nci I and sequencing of the long-range PCR product confirmed the absence of leu444pro in the mother. A western blot demonstrated normal expression of GCase in maternal cells, whereas the affected infant displayed markedly decreased GCase expression (Figure 1b). Finally, GCase activity assayed in the proband, mother and two controls confirmed that levels of activity in maternal samples were similar to controls, while the proband showed severely deficient GCase activity typical of type 2 GD (Figure 1c). Therefore, the results of GBA1 sequencing, restriction digests, western blots and GCase activity all provided no evidence of a somatic maternal mutation.

In proband no. 2, genomic sequencing of GBA1 demonstrated the genotype leu444pro/leu444pro + ala456pro (c.1483 G > C;p.ala495pro). His father was heterozygous for the leu444pro + ala456pro allele, but no mutation was detected in DNA samples from the a

The proband: T323I/L444P

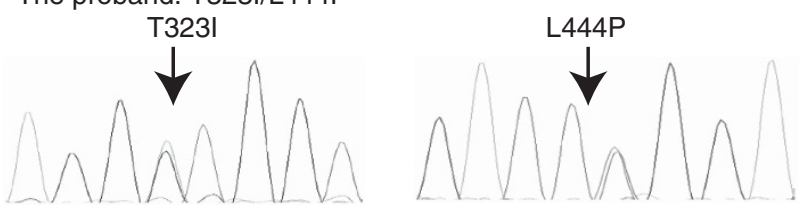

Father: WT/T323I

T323I

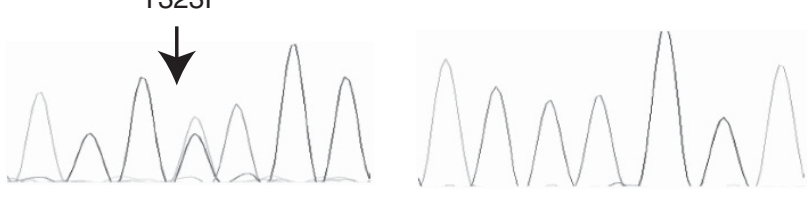

b

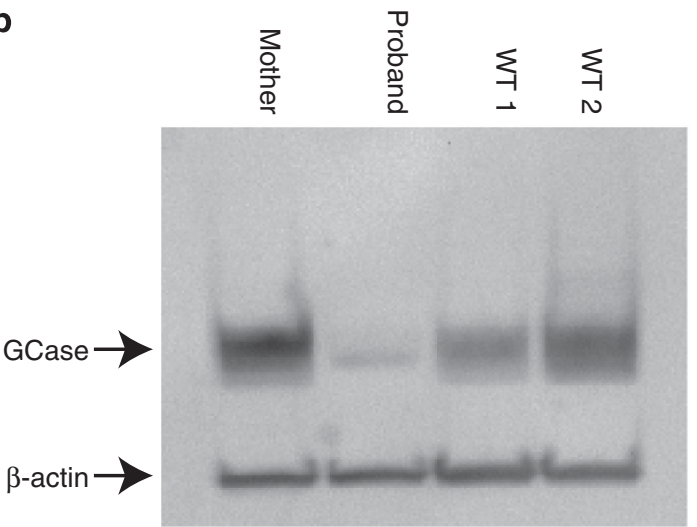

C

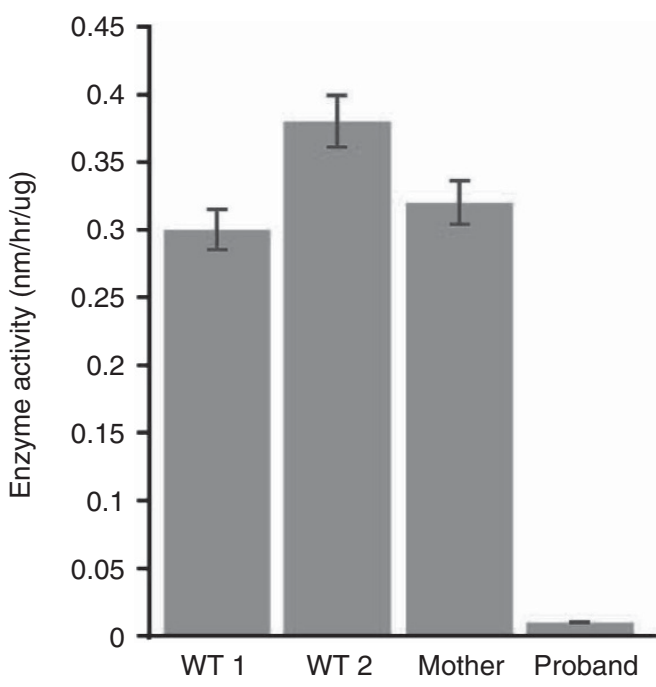

Figure 1 Studies in Family no. 1. (a) Chromatograms showing GBA1 sequence including the proband, mother and father. Each individual genotype has been labeled. (b) Western blot probed with antibody to GCase performed on total protein extracted from the proband, mother and two WT controls fibroblast cell lines. GCase levels were compared with $\beta$-actin. (c) GCase activity measured in fibroblast extracts from the proband, mother and two WT controls.

mother, sister or maternal grandmother. Maternity was confirmed with $99.7 \%$ certainty.

In both families, the hetrozygosity of polymorphic sites surrounding position c.1448 was confirmed by the complete amplification of 
the maternal GBA1 allele. In addition, sequencing of GBA1 amplified by long-range PCR confirmed that there were no deletions or nucleotide variations at the primer sites.

\section{DISCUSSION}

On the basis of results described, we propose that the leu444pro mutation in both families originated in the mothers' ova, either as a result of germline mosaicism or a de novo mutation in one ovum that took place during cell division (mitosis/meiosis). Multiple affected offspring from non-carrier parents is usually attributed to germline mosaicism. In our study, none of the other offspring was a carrier for leu444pro, enhancing the probability that the leu444pro mutation resulted from a de novo mutation rather than a germline mosaicism. Unfortunately, these possibilities cannot be definitively confirmed without ovarian biopsies. A less likely alternative possibility is that the mutation arose as an early post-zygotic event in the proband, leading to mosaicism. The high frequency of the leu444pro mutation among different populations, along with evidence that this site has been a mutational hotspot, could explain the nucleotide transversion originating at this site. Although common in different types of cancers, germline mutations are rare in autosomal disorders. There have been reports of germline mutations in several dominant disorders such as achondroplasia, Apert syndrome and multiple endocrine neoplasia, ${ }^{22}$ and in one patient with Ataxia Telangiectasia, an autosomal recessive disorder. ${ }^{23}$ However, this is the first report of the presence of the same maternal germline mutation in two unrelated families with GD, with significant implications for molecular diagnostics and genetic counseling. Such germline mutations may not always be evident, as parental DNA is not consistently evaluated. This finding has implications for genetic counseling, for if only one parent is a carrier for a recessive disorder, the chance of having an affected child may not be zero and the possibility of germline mosaicism or a de novo mutation should be considered. The identification of the two cases reported suggests that these events may occur more often than appreciated in GD and other recessive disorders.

\section{CONFLICT OF INTEREST}

The authors declare no conflict of interest.

\section{ACKNOWLEDGEMENTS}

This work was supported by the Intramural Research Programs of the National Human Genome Research Institute and National Institutes of Health. We thank Dr Suzanne Hart for her helpful suggestions.
1 Sidransky E: Gaucher disease: complexity in a 'simple' disorder. Mol Genet Metab 2004; 83: 6-15.

2 Beutler E, Grabowski G: Gaucher disease; in Scriver C, Beudet A, Sly W, Valle D (eds): The Metabolic and Molecular Bases of Inherited Disease. New York: McGraw-Hill, 1995; Vol 2, pp 2641-2670.

3 Hruska KS, LaMarca ME, Scott CR, Sidransky E: Gaucher disease: mutation and polymorphism spectrum in the glucocerebrosidase gene (GBA). Hum Mutat 2008; 29: 567-583.

4 Cormand B, Vilageliu L, Burguera JM et al: Gaucher disease in Spanish patients: analysis of eight mutations. Hum Mutat 1995; 5: 303-309.

5 Drugan C, Procopciuc L, Jebeleanu G et al: Gaucher disease in Romanian patients: incidence of the most common mutations and phenotypic manifestations. Eur J Hum Genet 2002; 10: 511-515.

6 Hodanova K, Hrebicek M, Cervenkova M, Mrazova L, Veprekova L, Zemen J: Analysis of the beta-glucocerebrosidase gene in Czech and Slovak Gaucher patients: mutation profile and description of six novel mutant alleles. Blood Cells Mol Dis 1999; 25: 287298.

7 Emre S, Gurakan F, Yuce A, Rolf A, Scott R, Ozen H: Molecular analysis of Turkish Gaucher disease patients: identification of novel mutations in glucocerebrosidase (GBA) gene. Eur J Med Genet 2008; 51: 315-321.

8 Wan L, Hsu CM, Tsai CH, Lee CC, Hwu WL, Tsai FJ: Mutation analysis of Gaucher disease patients in Taiwan: high prevalence of the RecNcil and L444P mutations. Blood Cells Mol Dis 2006; 36: 422-425.

9 Zhang WM, Tang NL, Meng Y et al: [An analysis of mutations causing Gaucher disease in Chinese population]. Zhonghua Yi Xue Za Zhi 2009; 89: 3397-3400.

10 Dahl N, Hillborg PO, Olofsson A: Gaucher disease (Norrbottnian type III): probable founders identified by genealogical and molecular studies. Hum Genet 1993; 92: $513-515$.

11 Tayebi N, Stubblefield BK, Park JK et al: Reciprocal and nonreciprocal recombination at the glucocerebrosidase gene region: implications for complexity in Gaucher disease. Am J Hum Genet 2003; 72: 519-534.

12 Arnheim N, Calabrese P: Understanding what determines the frequency and pattern of human germline mutations. Nat Rev Genet 2009; 10: 478-488.

13 Choi SK, Yoon SR, Calabrese P, Arnheim N: A germ-line-selective advantage rather than an increased mutation rate can explain some unexpectedly common human disease mutations. Proc Natl Acad Sci USA 2008; 105: 10143-10148.

14 Alfonso P, Pocovi M, Giraldo P: Gaucher disease: Report of de nova mutation in a Spanish family. Blood Cells Mol Dis 2008; 40: 444-445.

15 Aviner S, Garty BZ, Rachmel A et al: Type 2 Gaucher disease occurs in Ashkenazi Jews but is surprisingly rare. Blood Cells Mol Dis 2009; 43: 294-297.

16 Stone DL, Tayebi N, Orvisky E, Stubblefield B, Madike V, Sidransky E: Glucocerebrosidase gene mutations in patients with type 2 Gaucher disease. Hum Mutat 2000; 15: $181-188$.

17 Uchiyama A, Tomatsu S, Kondo $\mathrm{N}$ et al: New Gaucher disease mutations in exon 10: a novel L444R mutation produces a new Ncil site the same as L444P. Hum Mol Genet 1994; 3: 1183-1184.

18 Tayebi N, Cushner S, Sidransky E: Differentiation of the glucocerebrosidase gene from pseudogene by long-template PCR: implications for Gaucher disease. Am J Hum Genet 1996; 59: 740-741.

19 Velayati A, DePaolo J, Gupta N et al: A mutation in SCARB2 is a modifier in Gaucher disease. Hum Mutat 2011; 32: 1232-1238.

20 Raghavan SS, Topol J, Kolodny EH: Leukocyte beta-glucosidase in homozygotes and heterozygotes for Gaucher disease. Am J Hum Genet 1980; 32: 158-173.

21 He G-S, Grace M, Grabowski G: Gaucher disease: four rare alleles encoding F213।, P289L, T323I, and R463C in type 1 variants. Hum Mutat 1992; 1: 423-427.

22 Broeks A, Urbanus JH, Floore AN et al: ATM heterozygous germline mutations contribute to breast cancer-susceptibility. Am J Hum Genet 2000; 66: 494-500.

23 Broeks A, de Klein A, Floore AN et al: ATM germline mutations in classical ataxia-telangiectasia patients in the Dutch population. Hum Mutat 1998; 12: 330-337. 